\title{
Flux upper limits for 47 AGN observed with H.E.S.S. in 2004-2011
}

H.E.S.S. Collaboration, A. Abramowski ${ }^{1}$, F. Aharonian, ${ }^{2,3,4}$, F. Ait Benkhali ${ }^{2}$, A. G. Akhperjanian ${ }^{5,4}$, E. Angüner ${ }^{6}$, G. Anton ${ }^{7}$, S. Balenderan ${ }^{8}$, A. Balzer ${ }^{9,10}$, A. Barnacka ${ }^{11}$, Y. Becherini ${ }^{12}$, J. Becker Tjus ${ }^{13}$, K. Bernlöhr²,6, E. Birsin ${ }^{6}$, E. Bissaldi ${ }^{14}$, J. Biteau ${ }^{15,16, \star}$, M. Böttcher ${ }^{17}$, C. Boisson ${ }^{18}$, J. Bolmont ${ }^{19}$, P. Bordas ${ }^{20}$, J. Brucker ${ }^{7}$, F. Brun ${ }^{2}$, P. Brun ${ }^{21}$, T. Bulik ${ }^{22}$, S. Carrigan ${ }^{2}$, S. Casanova ${ }^{17,2}$, M. Cerruti ${ }^{18,23}$, P. M. Chadwick ${ }^{8}$, R. Chalme-Calvet ${ }^{19}$, R. C.G. Chaves ${ }^{21}$, A. Cheesebrough ${ }^{8}$, M. Chrétien ${ }^{19}$, S. Colafrancesco ${ }^{24}$, G. Cologna ${ }^{25}$, J. Conrad $^{26,27}$, C. Couturier ${ }^{19}$, Y. Cui $^{20}$, M. Dalton ${ }^{28,29}$, M. K. Daniel ${ }^{8}$, I. D. Davids ${ }^{17,30}$, B. Degrange ${ }^{15}$, C. Deil ${ }^{2}$, P. deWilt $^{31}$, H. J. Dickinson ${ }^{26}$, A. Djannati-Atai ${ }^{32}$, W. Domainko ${ }^{2}$, L.O'C. Drury ${ }^{3}$, G. Dubus ${ }^{33}$, K. Dutson ${ }^{34}$, J. Dyks ${ }^{11}$, M. Dyrda ${ }^{35}$, T. Edwards ${ }^{2}$,

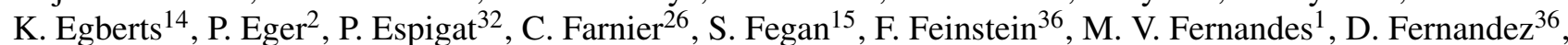
A. Fiasson ${ }^{37}$, G. Fontaine ${ }^{15}$, A. Förster ${ }^{2}$, M. Füßling ${ }^{10}$, M. Gajdus ${ }^{6}$, Y. A. Gallant ${ }^{36}$, T. Garrigoux ${ }^{19}$, G. Giavitto ${ }^{9}$, B. Giebels ${ }^{15}$, J. F. Glicenstein ${ }^{21}$, M.-H. Grondin ${ }^{2,25}$, M. Grudzińska ${ }^{22}$, S. Häffner ${ }^{7}$, J. Hahn ${ }^{2}$, J. Harris ${ }^{8}$,

G. Heinzelmann ${ }^{1}$, G. Henri ${ }^{33}$, G. Hermann ${ }^{2}$, O. Hervet ${ }^{18}$, A. Hillert ${ }^{2}$, J. A. Hinton ${ }^{34}$, W. Hofmann ${ }^{2}$, P. Hofverberg ${ }^{2}$, M. Holler ${ }^{10}$, D. Horns ${ }^{1}$, A. Jacholkowska ${ }^{19}$, C. Jahn ${ }^{7}$, M. Jamrozy ${ }^{38}$, M. Janiak ${ }^{11}$, F. Jankowsky ${ }^{25}$, I. Jung ${ }^{7}$, M. A. Kastendieck ${ }^{1}$, K. Katarzyński ${ }^{39}$, U. Katz ${ }^{7}$, S. Kaufmann²5, B. Khélifi ${ }^{32}$, M. Kieffer ${ }^{19}$, S. Klepser ${ }^{9}$, D. Klochkov ${ }^{20}$, W. Kluźniak ${ }^{11}$, T. Kneiske ${ }^{1}$, D. Kolitzus ${ }^{14}$, Nu. Komin ${ }^{37}$, K. Kosack ${ }^{21}$, S. Krakau ${ }^{13}$, F. Krayzel ${ }^{37}$, P. P. Krüger ${ }^{17,2}$, H. Laffon ${ }^{28}$, G. Lamanna ${ }^{37}$, J. Lefaucheur ${ }^{32}$, A. Lemière ${ }^{32}$, M. Lemoine-Goumard ${ }^{28}$, J.-P. Lenain ${ }^{19}$, D. Lennarz ${ }^{2}$, T. Lohse $^{6}$, A. Lopatin ${ }^{7}$, C.-C. Lu $^{2}$, V. Marandon ${ }^{2}$, A. Marcowith ${ }^{36}$, R. Marx ${ }^{2}$, G. Maurin ${ }^{37}$, N. Maxted ${ }^{31}$, M. Mayer ${ }^{10}$, T. J.L. McComb ${ }^{8}$, J. Méhault ${ }^{28,29}$, P. J. Meintjes ${ }^{40}$, U. Menzler ${ }^{13}$, M. Meyer ${ }^{26}$, R. Moderski ${ }^{11}$, M. Mohamed ${ }^{25}$, E. Moulin ${ }^{21}$, T. Murach ${ }^{6}$, C. L. Naumann ${ }^{19}$, M. de Naurois ${ }^{15}$, J. Niemiec ${ }^{35}$, S. J. Nolan ${ }^{8}$, L. Oakes ${ }^{6}$, S. Ohm ${ }^{34}$, E. de Oña Wilhelmi ${ }^{2}$, B. Opitz ${ }^{1}$, M. Ostrowski ${ }^{38}$, I. Oya ${ }^{6}$, M. Panter ${ }^{2}$, R. D. Parsons ${ }^{2}$, M. Paz Arribas ${ }^{6}$, N. W. Pekeur ${ }^{17}$, G. Pelletier ${ }^{33}$, J. Perez ${ }^{14}$, P.-O. Petrucci ${ }^{33}$, B. Peyaud ${ }^{21}$, S. Pita ${ }^{32}$, H. Poon ${ }^{2}$, G. Pühlhofer ${ }^{20}$, M. Punch ${ }^{32}$, A. Quirrenbach ${ }^{25}$, S. Raab $^{7}$, M. Raue ${ }^{1}$, A. Reimer ${ }^{14}$, O. Reimer ${ }^{14}$, M. Renaud $^{36}$, R. de los Reyes ${ }^{2}$, F. Rieger ${ }^{2}$, L. Rob ${ }^{41}$, C. Romoli ${ }^{3}$, S. Rosier-Lees ${ }^{37}$, G. Rowell ${ }^{31}$, B. Rudak ${ }^{11}$, C. B. Rulten ${ }^{18}$, V. Sahakian ${ }^{5,4}$, D. A. Sanchez $2,37, \star$, A. Santangelo ${ }^{20}$, R. Schlickeiser ${ }^{13}$, F. Schüssler ${ }^{21}$, A. Schulz ${ }^{9}$, U. Schwanke ${ }^{6}$, S. Schwarzburg ${ }^{20}$, S. Schwemmer ${ }^{25}$, H. Sol ${ }^{18}$, G. Spengler ${ }^{6}$, F. Spies ${ }^{1}$, Ł. Stawarz ${ }^{38}$, R. Steenkamp ${ }^{30}$, C. Stegmann ${ }^{10,9}$, F. Stinzing ${ }^{7}$, K. Stycz ${ }^{9}$, I. Sushch ${ }^{6,17}$, A. Szostek ${ }^{38}$, J.-P. Tavernet ${ }^{19}$, T. Tavernier ${ }^{32}$, A. M. Taylor ${ }^{3}$, R. Terrier ${ }^{32}$, M. Tluczykont ${ }^{1}$, C. Trichard ${ }^{37}$, K. Valerius ${ }^{7}$, C. van Eldik ${ }^{7}$, B. van Soelen ${ }^{40}$, G. Vasileiadis ${ }^{36}$, C. Venter ${ }^{17}$, A. Viana ${ }^{2}$, P. Vincent ${ }^{19}$, H. J. Völk ${ }^{2}$, F. Volpe ${ }^{2}$, M. Vorster ${ }^{17}$, T. Vuillaume ${ }^{33}$, S. J. Wagner ${ }^{25}$, P. Wagner ${ }^{6}$, M. Ward ${ }^{8}$, M. Weidinger ${ }^{13}$, Q. Weitzel ${ }^{2}$, R. White ${ }^{34}$, A. Wierzcholska ${ }^{38}$, P. Willmann ${ }^{7}$, A. Wörnlein ${ }^{7}$, D. Wouters ${ }^{21}$, V. Zabalza ${ }^{2}$, M. Zacharias ${ }^{13}$, A. Zajczyk ${ }^{11,36}$, A. A. Zdziarski ${ }^{11}$, A. Zech ${ }^{18}$, and H.-S. Zechlin ${ }^{1}$

(Affiliations can be found after the references)

Received 22 October 2013 / Accepted 5 February 2014

\section{ABSTRACT}

Context. About $40 \%$ of the observation time of the High Energy Stereoscopic System (H.E.S.S.) is dedicated to studying active galactic nuclei (AGN), with the aim of increasing the sample of known extragalactic very-high-energy (VHE, $E>100 \mathrm{GeV}$ ) sources and constraining the physical processes at play in potential emitters.

Aims. H.E.S.S. observations of AGN, spanning a period from April 2004 to December 2011, are investigated to constrain their $\gamma$-ray fluxes. Only the 47 sources without significant excess detected at the position of the targets are presented.

Methods. Upper limits on VHE fluxes of the targets were computed and a search for variability was performed on the nightly time scale.

Results. For 41 objects, the flux upper limits we derived are the most constraining reported to date. These constraints at VHE are compared with the flux level expected from extrapolations of Fermi-LAT measurements in the two-year catalog of AGN. The H.E.S.S. upper limits are at least a factor of two lower than the extrapolated Fermi-LAT fluxes for 11 objects. Taking into account the attenuation by the extragalactic background light reduces the tension for all but two of them, suggesting intrinsic curvature in the high-energy spectra of these two AGN.

Conclusions. Compilation efforts led by current VHE instruments are of critical importance for target-selection strategies before the advent of the Cherenkov Telescope Array (CTA).

Key words. gamma rays: galaxies - galaxies: active

\footnotetext{
* Corresponding authors: J. Biteau, e-mail: biteau@in2p3.fr; D.A. Sanchez, e-mail: david.sanchez@lapp.in2p3.fr
} 


\section{Introduction}

Since the discovery of their extragalactic origin fifty years ago (Schmidt 1963), the class of astrophysical sources called active galactic nuclei (AGN) has been a prime target for astronomers that observe the sky from radio wavelengths to very-high-energy $\gamma$ rays (VHE, $E>100 \mathrm{GeV}$ ). AGN are thought to host supermassive black holes (typical mass of $10^{8-9} M_{\odot}$ ) surrounded by an accretion disk, with a fraction of them showing two-sided jets. To unify the various subclasses of AGN, a scheme to sort them based on their orientation with respect to the observer's line of sight has been proposed since the 1990s (Urry \& Padovani 1995). Objects whose jets are closely aligned with the line of sight are called blazars. They fall into two source classes, broad-line-spectrum sources called flat spectrum radio quasars (FSRQs), and BL Lac objects (hereafter BL in tables), which show faint lines or featureless spectra.

Active galactic nuclei, in particular blazars, are the most numerous objects detected at high energy (HE, $100 \mathrm{MeV}<$ $E<100 \mathrm{GeV}$ ), where all-sky surveys can be performed with pair-conversion detectors onboard satellites such as the Fermi Large Area Telescope (LAT, Atwood et al. 2009). The Second LAT AGN Catalog, hereafter 2LAC, comprises 886 off-plane (i.e. above a Galactic latitude of $10^{\circ}$ ) point-like sources associated with AGN that were detected in two years of operation (Ackermann et al. 2011). AGN constitute a third of the sources known at VHE, despite a coverage biased toward Galactic sources. With the fast decrease of fluxes with increasing energy, observations at VHE are mostly performed with ground-based imaging atmospheric Cherenkov telescopes (IACT), which have a field of view (FoV) of a few degrees but an effective area on the order of $10^{5} \mathrm{~m}^{2}$. Their current sensitivity prevents an all-sky scan in a reasonable amount of time, and IACT observations must be pointed to targets of interest (see e.g. Dubus et al. 2013 for a discussion of the capabilities of next generation instruments). Targeted AGN are selected based on their radio and X-ray spectra (Stecker et al. 1996; Perlman 2000; Costamante \& Ghisellini 2002) as well as based on their HE flux extrapolated to VHE (Tavecchio et al. 2010).

The High Energy Stereoscopic System (H.E.S.S., Aharonian et al. 2006a) has significantly contributed to the expansion of the class of VHE AGN, with the detection of 23 objects, including 20 discoveries, among 56 known sources of this type as of the end of the year $2013^{1}$. The H.E.S.S. experiment is located in the Khomas Highland, Namibia $\left(23^{\circ} 16^{\prime} 18^{\prime \prime} \mathrm{S}, 16^{\circ} 30^{\prime} 01^{\prime \prime} \mathrm{E}\right)$ at an altitude of $1800 \mathrm{~m}$ above sea level. In its first phase, this experiment was an array of four identical telescopes with cameras composed of 960 photomultipliers and segmented reflectors paving a reflective area of $107 \mathrm{~m}^{2}$, for an equivalent diameter of $12 \mathrm{~m}$. Most of the AGN detected with H.E.S.S. belong to the BL Lac class, as shown in Table 1, with the exception of the two nearby radio galaxies of Fanaroff-Riley I type (FR I) Centaurus A and M 87, the FSRQ PKS 1510-089, and the blazar candidate HESS J1943+213, which is located in the Galactic plane. In addition to constraining the radiative processes responsible for the $\gamma$-ray emission (for detailed discussions, see, e.g., the references in Table 1), the VHE spectra of these objects can also serve cosmological purposes, as shown with the constraints (Aharonian et al. 2006b; Mazin \& Raue 2007) and indirect measurement (Abramowski et al. 2013d) of the extragalactic background light (EBL). With peak intensities in the optical and farinfrared bands, the EBL is composed of the integrated emission

TeVCat, http://tevcat. uchicago.edu/ of stars and galaxies as well as of the reprocessing of UV-tooptical light by dust. The EBL can hardly be measured directly, although it is the second-most intense diffuse radiation in the Universe after the cosmic microwave background.

During the eight years of the first phase of H.E.S.S., some of the observations did not result in significant excesses at the position of the target or in the FoV of the telescopes. A first set of upper limits (Aharonian et al. 2005b, hereafter HUL1) on 19 AGN observed during 63 hours was published after two years of observation. A second paper (Aharonian et al. 2008b, hereafter HUL2) listed 14 upper limits based on 94 hours of observation spanning 2005-2007. In this paper, which follows extensive compilation efforts from previous-generation instruments such as Whipple (Horan et al. 2004) or HEGRA (Aharonian et al. 2004), 47 selected candidates are studied, with observations spanning April 2004 to December 2011, for a total live time of approximately $400 \mathrm{~h}$. The candidates and the data selection are presented in Sect. 2, the event and spectral analyses are examined in Sect. 3, and the constraints on the VHE emission are discussed in Sect. 4, together with the target-selection strategy.

\section{Selected candidate VHE emitters}

The sample of targets consists of the AGN observed with H.E.S.S. until December 2011, for which more than an hour of corrected live time was recorded (see Sect. 3). Only objects located away from the Galactic plane, that is above a Galactic latitude of $10^{\circ}$, were taken into account. Neither datasets on potential or detected H.E.S.S. sources ${ }^{2}$ are included, nor those where upper limits based on the entire dataset have already been published (HUL1, HUL2). The objects listed in the 2LAC that are located in the same FoV as selected targets and are potentially associated with AGN were also studied. These criteria yield a total of 42 AGN and 5 unknown-type Fermi-LAT or EGRET sources, as listed in Table 2. Pointed observations were performed for 33 objects, while 14 are visible in the FoVs. Of these 47 targets, 39 are studied for the first time with H.E.S.S. in this paper, while eight of them (IIIZw 2, 1ES 0323+022, 3C 120, Pictor A, 1ES 1440+122, RBS 1888, NGC 7469, 1ES 2343-151) have been re-observed since the publication of HUL1 and HUL2.

The redshifts of the targets were extracted from the RomaBZCAT catalog Ed. 4.1.1 (Massaro et al. 2009), from the work of Rau et al. (2012) on AGN detected with Fermi-LAT, and from the publication by Pita et al. (2012) about VHE (candidate) emitters. The redshifts of objects not listed in these publications were individually searched for in the literature. A detailed list of references can be found in the last column of Table 2. The distant objects with $z>1$ in this table were not directly targeted. 2FGL J0426.6+0509c is located in the same FoV as 3C 120. 2FGL J1959.1-4245 and 2FGL J2219.1+1805 are neighbors of PKS 2004-447 and RBS 1888, respectively. 2FGL J0505.80411 and 2FGL J0540.4-5415 were jointly observed with BZB J0543-5532.

The classification of the targets is also primarily based on BZCAT. For objects not listed there, the 2LAC catalog was followed, yielding two AGN of unknown type in addition to BL Lac and FSRQ objects: 1FGL J0506.9-5435 and 2FGL J0537.7-5716, called AGU in Table 2, following the

\footnotetext{
2 A source is considered as detected above a significance of $5 \sigma$, while a potential source corresponds to an extrapolated observation time needed to reach detection shorter than $40 \mathrm{~h}$. The list of objects studied in this paper does not depend on the latter criterion within $\pm 10 \mathrm{~h}$.
} 
Table 1. AGN detected by H.E.S.S. as of September 2013.

\begin{tabular}{lccc}
\hline \hline Object & $z$ & Type & Reference \\
\hline Cen A & 0.002 & FR I & Aharonian et al. (2009) \\
M 87 & 0.004 & FR I & Abramowski et al. (2012a) \\
Markarian 421 & 0.031 & BL & Aharonian et al. (2005a) \\
AP Librae & 0.049 & BL & Sanchez et al. (2012) \\
PKS 1440-389 & 0.065 & BL & Hofmann (2012) \\
PKS 0548-322 & 0.069 & BL & Aharonian et al. (2010) \\
PKS 2005-489 & 0.071 & BL & Abramowski et al. (2011b) \\
RGB J0152+017 & 0.080 & BL & Aharonian et al. (2008c) \\
SHBL J001355.9-185406 & 0.095 & BL & Abramowski et al. (2013b) \\
1ES 1312-423 & 0.105 & BL & Abramowski et al. (2013f) \\
PKS 2155-304 & 0.116 & BL & Abramowski et al. (2012c) \\
1ES 0229+200 & 0.140 & BL & Aharonian et al. (2007a) \\
1RXS J101015.9-311909 & 0.143 & BL & Abramowski et al. (2012d) \\
H 2356-309 & 0.165 & BL & Abramowski et al. (2010) \\
1ES 1101-232 & 0.186 & BL & Aharonian et al. (2007c) \\
1ES 0347-121 & 0.188 & BL & Aharonian et al. (2007b) \\
PKS 0301-243 & 0.266 & BL & Abramowski et al. (2013a) \\
1ES 0414+009 & 0.287 & BL & Abramowski et al. (2012b) \\
PKS 1510-089 & 0.361 & FSRQ & Abramowski et al. (2013c) \\
PKS 0447-439 & $<0.57^{a}$ & BL & Abramowski et al. (2013e) \\
PG 1553+113 & - & BL & Aharonian et al. (2008a) \\
HESS J1943+213 & - & - & Abramowski et al. (2011a) \\
KUV 00311-1938 & $>0.506^{b}$ & BL & Becherini et al. (2012) \\
\hline
\end{tabular}

Notes. The redshift, classification and latest H.E.S.S. publication on the source are given in Cols. 2 to 4 . Acronyms are defined in the text.

References. ${ }^{(a)}$ See Pita et al. (2012). ${ }^{(b)}$ See Abramowski et al. (2013e).

2LAC nomenclature. Following HUL1 and HUL2, 3C 120 and Pictor A are classified as Fanaroff-Riley I (FR I) and II (FR II) radio galaxies. Searching the SIMBAD database ${ }^{3}$, Seyfert 1 nuclei (Sey I) are hosted by these two objects and by NGC 7469, while PKS $1345+125$ is classified as a Seyfert 2 (Sey II). To summarize, most of the targets are blazars, with 13 FSRQ and $23 \mathrm{BL}$ Lac objects, including PKS 0352-686 and 1FGL J0022.2-1850 recently confirmed as BL Lac objects by Rodriguez et al. (2009) and Shaw et al. (2013), respectively.

\section{Analysis and results}

The observation conditions and the results of the event analyses are listed in Table 3. The H.E.S.S. telescopes are usually pointed with an offset angle of $0.5-0.7^{\circ}$ (wobble mode) when observing extragalactic sources. Higher offset values occur in Table 3 because sources can be in the same FoV as a scheduled target source. The comparably (with other IACTs) large FoV of $5^{\circ}$ of H.E.S.S. telescopes allows for reliable spectral reconstruction up to an offset of at least $2^{\circ}$ (offset value in Abramowski et al. 2013f), close to the maximum offset values that are listed in Table 3. The observation time, shown in the second column, is corrected for the decrease in acceptance due to an increasing offset from the centre of the cameras. This correction results in a shorter acceptance-corrected live time, as shown in Col. 5.

The data that pass standard quality criteria (good weather, stability of the instrument, as in Aharonian et al. 2006a) were analyzed with Model++ Standard Cuts (de Naurois \& Rolland 2009), corresponding to a selection criterion on the image charge of 60 photo electrons. A cross-check was performed with a

\footnotetext{
3 http://simbad.u-strasbg.fr/simbad/
}

multivariate analysis described in Ohm et al. (2009). The results of the analysis of the 47 targets described in the following were derived with a single pipeline, associated to the Model ++ analysis. The analysis energy threshold ${ }^{4}$, shown in Col. 6, depends on the average zenith angle of the observations (Col. 3) and on the offset from the center of the cameras (Col. 4). The number of ON-target (Col. 7) and OFF-target events (Col. 8) was measured above the threshold energy in regions of $0.1^{\circ}$ radius. The normalization $\alpha$ of the OFF events, shown in Col. 9, is a relative exposure normalization factor between the $\mathrm{ON}$ and OFF regions, within the Reflected background modeling method (Aharonian et al. 2006a; Berge et al. 2007). The excess, defined as $\mathrm{ON}-\alpha \times \mathrm{OFF}$, and its significance, calculated using Eq. (17) in Li \& Ma (1983), are shown in the last two columns of Table 3. No significant deviation from zero is observed, with values in the range $[-2.2 \sigma ; 2.4 \sigma]$.

The distribution of the detection significance is compared in Fig. 1 with a normal distribution of 47 events, centered on zero and of unitary standard deviation. The deviations of the data distribution from the normal distribution were quantified using a Kolmogorov-Smirnov test. The highest value of the absolute difference between the cumulative probability distributions reaches 0.17 , with a $p$-value for a normal distribution of $12 \%$, equivalent to a 1.5 Gaussian standard deviation. An AndersonDarling test yields a similar result, with a $p$-value for a normal

4 Hereafter, the energy threshold is defined as the energy for which the acceptance reaches $20 \%$ of the highest value. This approach, which results in a somewhat lower threshold than the conventional definition (peak of the energy distribution of the events), corresponds to an energy bias lower than the energy resolution (see Figs. 23, 24 in de Naurois \& Rolland 2009), which ensures the quality of the reconstructed spectrum. 
A\&A 564, A9 (2014)

Table 2. Selected extragalactic objects observed with H.E.S.S. from April 2004 to December 2011.

\begin{tabular}{|c|c|c|c|c|c|}
\hline Object & $\alpha_{\mathrm{J} 2000}$ & $\delta_{\mathrm{J} 2000}$ & $z$ & Type & Redshift reference \\
\hline IIIZw 2 & $00^{\mathrm{h}} 10^{\mathrm{m}} 31.2^{\mathrm{s}}$ & $+10^{\circ} 58^{\prime} 12^{\prime \prime}$ & 0.09 & FSRQ & Hernán-Caballero \& Hatziminaoglou (2011) \\
\hline 1FGL J0022.2-1850 & $00^{\mathrm{h}} 22^{\mathrm{m}} 16.8^{\mathrm{s}}$ & $-18^{\circ} 51^{\prime} 00^{\prime \prime}$ & $\begin{array}{l}>0.77 \\
<1.38\end{array}$ & $\mathrm{BL}$ & $\begin{array}{l}\text { Shaw et al. (2013) } \\
\text { Rau et al. (2012) }\end{array}$ \\
\hline 2FGL J0048.8-6347 & $00^{\mathrm{h}} 48^{\mathrm{m}} 52.8^{\mathrm{s}}$ & $-63^{\circ} 48^{\prime} 00^{\prime \prime}$ & - & - & - \\
\hline PKS 0048-097 & $00^{\mathrm{h}} 50^{\mathrm{m}} 40.8^{\mathrm{s}}$ & $-09^{\circ} 28^{\prime} 48^{\prime \prime}$ & 0.64 & BL & Rau et al. (2012) \\
\hline 1FGL J0051.4-6242 & $00^{\mathrm{h}} 51^{\mathrm{m}} 31.2^{\mathrm{s}}$ & $-62^{\circ} 42^{\prime} 36^{\prime \prime}$ & $<1.12$ & BL & Rau et al. (2012) \\
\hline RGB J0109+182 & $01^{\mathrm{h}} 09^{\mathrm{m}} 07.2^{\mathrm{s}}$ & $+18^{\circ} 16^{\prime} 12^{\prime \prime}$ & 0.14 & BL & Bauer et al. (2000) \\
\hline 2FGL J0211.2+1050 & $02^{\mathrm{h}} 11^{\mathrm{m}} 14.4^{\mathrm{s}}$ & $+10^{\circ} 50^{\prime} 24^{\prime \prime}$ & 0.20 & BL & Meisner \& Romani (2010) \\
\hline 2EG J0216+1107 & $02^{\mathrm{h}} 16^{\mathrm{m}} 00.0^{\mathrm{s}}$ & $+11^{\circ} 07^{\prime} 12^{\prime \prime}$ & - & - & - \\
\hline 2FGL J0229.3-3644 & $02^{\mathrm{h}} 29^{\mathrm{m}} 21.6^{\mathrm{s}}$ & $-36^{\circ} 43^{\prime} 48^{\prime \prime}$ & 2.12 & FSRQ & Hook et al. (2003) \\
\hline RBS 334 & $02^{\mathrm{h}} 37^{\mathrm{m}} 33.6^{\mathrm{s}}$ & $-36^{\circ} 03^{\prime} 36^{\prime \prime}$ & $0.41^{a}$ & $\mathrm{BL}$ & Pita et al. (2012) \\
\hline RBS 0413 & $03^{\mathrm{h}} 19^{\mathrm{m}} 52.8^{\mathrm{s}}$ & $+18^{\circ} 45^{\prime} 36^{\prime \prime}$ & 0.19 & BL & Donato et al. (2001) \\
\hline RBS 421 & $03^{\mathrm{h}} 25^{\mathrm{m}} 40.8^{\mathrm{s}}$ & $-16^{\circ} 46^{\prime} 12^{\prime \prime}$ & 0.29 & BL & Bauer et al. (2000) \\
\hline 1ES $0323+022$ & $03^{\mathrm{h}} 26^{\mathrm{m}} 14.4^{\mathrm{s}}$ & $+02^{\circ} 25^{\prime} 12^{\prime \prime}$ & 0.15 & BL & Laurent-Muehleisen et al. (1999) \\
\hline QSO B0331-362 & $03^{\mathrm{h}} 33^{\mathrm{m}} 12.0^{\mathrm{s}}$ & $-36^{\circ} 19^{\prime} 48^{\prime \prime}$ & 0.31 & BL & Woo et al. (2005) \\
\hline 2FGL J0334.3-3728 & $03^{\mathrm{h}} 34^{\mathrm{m}} 19.2^{\mathrm{s}}$ & $-37^{\circ} 28^{\prime} 12^{\prime \prime}$ & $<1.34$ & BL & Rau et al. (2012) \\
\hline PKS 0352-686 & $03^{\mathrm{h}} 52^{\mathrm{m}} 57.6^{\mathrm{s}}$ & $-68^{\circ} 31^{\prime} 12^{\prime \prime}$ & 0.09 & BL & Lavaux \& Hudson (2011) \\
\hline 2FGL J0426.6+0509c & $04^{\mathrm{h}} 26^{\mathrm{m}} 40.8^{\mathrm{s}}$ & $+05^{\circ} 09^{\prime} 00^{\prime \prime}$ & 1.33 & FSRQ & Kovalev et al. (1999) \\
\hline $3 \mathrm{C} 120$ & $04^{\mathrm{h}} 33^{\mathrm{m}} 12.0^{\mathrm{s}}$ & $+05^{\circ} 21^{\prime} 00^{\prime \prime}$ & 0.03 & FR I & Lavaux \& Hudson (2011) \\
\hline 2FGL J0505.8-0411 & $05^{\mathrm{h}} 05^{\mathrm{m}} 48.0^{\mathrm{s}}$ & $-04^{\circ} 12^{\prime} 00^{\prime \prime}$ & 1.48 & FSRQ & Barkhouse \& Hall (2001) \\
\hline 1FGL J0506.9-5435 & $05^{\mathrm{h}} 06^{\mathrm{m}} 57.6^{\mathrm{s}}$ & $-54^{\circ} 36^{\prime} 00^{\prime \prime}$ & $<1.07$ & AGU & Rau et al. (2012) \\
\hline 1ES 0507-040 & $05^{\mathrm{h}} 09^{\mathrm{m}} 38.4^{\mathrm{s}}$ & $-04^{\circ} 00^{\prime} 36^{\prime \prime}$ & 0.31 & BL & Woo et al. (2005) \\
\hline 2FGL J0515.0-4411 & $05^{\mathrm{h}} 15^{\mathrm{m}} 00.0^{\mathrm{s}}$ & $-44^{\circ} 12^{\prime} 00^{\prime \prime}$ & - & - & - \\
\hline 2FGL J0516.5-4601 & $05^{\mathrm{h}} 16^{\mathrm{m}} 33.6^{\mathrm{s}}$ & $-46^{\circ} 01^{\prime} 12^{\prime \prime}$ & 0.19 & FSRQ & Landt et al. (2004) \\
\hline Pictor A & $05^{\mathrm{h}} 19^{\mathrm{m}} 50.4^{\mathrm{s}}$ & $-45^{\circ} 46^{\prime} 48^{\prime \prime}$ & 0.03 & FR II & Liu \& Zhang (2002) \\
\hline 2FGL J0537.7-5716 & $05^{\mathrm{h}} 37^{\mathrm{m}} 43.2^{\mathrm{s}}$ & $-57^{\circ} 16^{\prime} 12^{\prime \prime}$ & 1.55 & AGU & Rau et al. (2012) \\
\hline 2FGL J0540.4-5415 & $05^{\mathrm{h}} 40^{\mathrm{m}} 26.4^{\mathrm{s}}$ & $-54^{\circ} 15^{\prime} 00^{\prime \prime}$ & 1.19 & FSRQ & Healey et al. (2008) \\
\hline BZB J0543-5532 & $05^{\mathrm{h}} 43^{\mathrm{m}} 57.6^{\mathrm{s}}$ & $-55^{\circ} 31^{\prime} 48^{\prime \prime}$ & 0.27 & $\mathrm{BL}$ & Pita et al. (2012) \\
\hline 1ES 0715-259 & $07^{\mathrm{h}} 18^{\mathrm{m}} 04.8^{\mathrm{s}}$ & $-26^{\circ} 08^{\prime} 24^{\prime \prime}$ & 0.47 & $\mathrm{BL}$ & Carangelo et al. (2003) \\
\hline RBS 1049 & $11^{\mathrm{h}} 54^{\mathrm{m}} 04.8^{\mathrm{s}}$ & $-00^{\circ} 10^{\prime} 12^{\prime \prime}$ & 0.25 & $\mathrm{BL}$ & Adelman-McCarthy \& et al. (2008) \\
\hline 1ES $1218+30.4$ & $12^{\mathrm{h}} 21^{\mathrm{m}} 21.6^{\mathrm{s}}$ & $+30^{\circ} 10^{\prime} 48^{\prime \prime}$ & 0.18 & BL & Adelman-McCarthy et al. (2009) \\
\hline 2FGL J1226.0+2953 & $12^{\mathrm{h}} 26^{\mathrm{m}} 04.8^{\mathrm{s}}$ & $+29^{\circ} 54^{\prime} 00^{\prime \prime}$ & - & - & - \\
\hline $3 \mathrm{C} 279$ & $12^{\mathrm{h}} 56^{\mathrm{m}} 12.0^{\mathrm{s}}$ & $-05^{\circ} 47^{\prime} 24^{\prime \prime}$ & 0.54 & FSRQ & Beckmann et al. (2006) \\
\hline 1ES $1332-295$ & $13^{\mathrm{h}} 35^{\mathrm{m}} 28.8^{\mathrm{s}}$ & $-29^{\circ} 50^{\prime} 24^{\prime \prime}$ & 0.26 & $\mathrm{BL}$ & Jones et al. (2009) \\
\hline PKS $1345+125$ & $13^{\mathrm{h}} 47^{\mathrm{m}} 33.6^{\mathrm{s}}$ & $+12^{\circ} 17^{\prime} 24^{\prime \prime}$ & 0.12 & Sey II & Adelman-McCarthy et al. (2009) \\
\hline 2FGL J1351.4+1115 & $13^{\mathrm{h}} 51^{\mathrm{m}} 28.8^{\mathrm{s}}$ & $+11^{\circ} 15^{\prime} 36^{\prime \prime}$ & 0.40 & $\mathrm{BL}$ & Adelman-McCarthy et al. (2009) \\
\hline 1ES $1440+122$ & $14^{\mathrm{h}} 42^{\mathrm{m}} 48.0^{\mathrm{s}}$ & $+12^{\circ} 00^{\prime} 36^{\prime \prime}$ & 0.16 & BL & Carangelo et al. (2003) \\
\hline 2FGL J1959.1-4245 & $19^{\mathrm{h}} 59^{\mathrm{m}} 09.6^{\mathrm{s}}$ & $-42^{\circ} 45^{\prime} 36^{\prime \prime}$ & 2.17 & FSRQ & Ghisellini et al. (2011) \\
\hline PKS 2004-447 & $20^{\mathrm{h}} 07^{\mathrm{m}} 55.2^{\mathrm{s}}$ & $-44^{\circ} 34^{\prime} 48^{\prime \prime}$ & 0.24 & FSRQ & Massaro et al. (2009) \\
\hline RBS 1752 & $21^{\mathrm{h}} 31^{\mathrm{m}} 36.0^{\mathrm{s}}$ & $-09^{\circ} 15^{\prime} 36^{\prime \prime}$ & 0.45 & $\mathrm{BL}$ & Giommi et al. (2005) \\
\hline PG $2209+184$ & $22^{\mathrm{h}} 11^{\mathrm{m}} 52.8^{\mathrm{s}}$ & $+18^{\circ} 42^{\prime} 00^{\prime \prime}$ & 0.07 & FSRQ & Paturel et al. (2002) \\
\hline 2 FGL J2219.1+1805 & $22^{\mathrm{h}} 19^{\mathrm{m}} 12.0^{\mathrm{s}}$ & $+18^{\circ} 05^{\prime} 24^{\prime \prime}$ & 1.80 & FSRQ & Sowards-Emmerd et al. (2005) \\
\hline RBS 1888 & $22^{\mathrm{h}} 43^{\mathrm{m}} 43.2^{\mathrm{s}}$ & $-12^{\circ} 31^{\prime} 12^{\prime \prime}$ & 0.23 & $\mathrm{BL}$ & Fischer et al. (1998) \\
\hline 3 EG J2248+1745 & $22^{\mathrm{h}} 48^{\mathrm{m}} 57.6^{\mathrm{s}}$ & $+17^{\circ} 46^{\prime} 12^{\prime \prime}$ & - & - & - \\
\hline NGC 7469 & $23^{\mathrm{h}} 03^{\mathrm{m}} 16.8^{\mathrm{s}}$ & $+08^{\circ} 52^{\prime} 12^{\prime \prime}$ & 0.02 & Sey I & Falco et al. (1999) \\
\hline PMN J2345-1555 & $23^{\mathrm{h}} 45^{\mathrm{m}} 12.0^{\mathrm{s}}$ & $-15^{\circ} 55^{\prime} 12^{\prime \prime}$ & 0.62 & FSRQ & Healey et al. (2008) \\
\hline 1ES 2343-151 & $23^{\mathrm{h}} 45^{\mathrm{m}} 38.4^{\mathrm{s}}$ & $-14^{\circ} 49^{\prime} 12^{\prime \prime}$ & 0.22 & $\mathrm{BL}$ & Schachter et al. (1993) \\
\hline 2FGL J2347.9-1629 & $23^{\mathrm{h}} 47^{\mathrm{m}} 55.2^{\mathrm{s}}$ & $-16^{\circ} 29^{\prime} 24^{\prime \prime}$ & 0.58 & FSRQ & Paturel et al. (2002) \\
\hline
\end{tabular}

Notes. Acronyms are defined in the text. ${ }^{(a)}$ Potential systematic uncertainties on the redshift of RBS 334 are discussed in Pita et al. (2012).

distribution of $10 \%$. These tests do not indicate a collective excess of events within the sample of source candidates.

As in HUL1 and HUL2, the spectral analysis was performed assuming a power-law spectrum with photon index $\Gamma=3$, close to values observed for the sources listed for instance in Table 1. Upper limits on the integral fluxes above the threshold energies were computed at the $99.9 \%$ confidence level, according to the statistical method of Rolke et al. (2005). The limits shown in Col. 4 of Table 4 were converted into Crab units (C.U., Col. 5) using the power-law spectrum measured by
Aharonian et al. (2006a), with a photon index $\Gamma=2.63$ and flux at $1 \mathrm{TeV} \phi_{0}=3.45 \times 10^{-11} \mathrm{~cm}^{-2} \mathrm{~s}^{-1} \mathrm{TeV}^{-1}$.

A search for variability, one of the characteristic properties of AGN, was performed by fitting a constant function to the flux estimates derived on a night-by-night time scale, as in HUL1 and HUL2. The modified Julian dates of observation for which at least one $\mathrm{ON}$-event is recorded are given in the last column of Table 4 and the $\chi^{2}$ probabilities for a constant fit (with $N_{\text {nights }}-1$ degrees of freedom) are shown in Col. 5. With $\chi^{2}$ probabilities higher than $10 \%$, no flaring event is detected on the nightly time 
H.E.S.S. Collaboration: Flux upper limits for 47 AGN

Table 3. Results from H.E.S.S. observations of 47 AGN.

\begin{tabular}{|c|c|c|c|c|c|c|c|c|c|c|}
\hline Object & $\begin{array}{c}T \\
{[\mathrm{~h}]}\end{array}$ & $Z_{\substack{\mathrm{obs} \\
\mathrm{o}}}$ & $\underset{\circ}{\text { Offset }}$ & $\begin{array}{r}T \text { (corr.) } \\
{[\mathrm{h}]} \\
\end{array}$ & $\begin{array}{c}E_{\mathrm{th}} \\
{[\mathrm{TeV}]}\end{array}$ & $\mathrm{ON}$ & OFF & $\alpha$ & Excess & $\begin{array}{c}S \\
{[\sigma]}\end{array}$ \\
\hline IIIZw 2 & 13.1 & 37 & 0.5 & 12.0 & 0.39 & 51 & 633 & 0.083 & -1.7 & -0.2 \\
\hline 1FGL J0022.2-1850 & 61.5 & 13 & 2.1 & 15.4 & 0.24 & 104 & 6348 & 0.018 & -13.1 & -1.2 \\
\hline 2FGL J0048.8-6347 & 8.0 & 40 & 1.2 & 4.9 & 0.58 & 23 & 431 & 0.033 & 8.8 & 2.1 \\
\hline PKS 0048-097 & 44.3 & 19 & 1.9 & 14.8 & 0.26 & 76 & 3418 & 0.023 & -3.2 & -0.4 \\
\hline 1FGL J0051.4-6242 & 8.0 & 40 & 0.5 & 7.4 & 0.58 & 10 & 193 & 0.083 & -6.1 & -1.6 \\
\hline RGB J0109+182 & 4.1 & 42 & 0.5 & 3.8 & 0.71 & 10 & 144 & 0.083 & -2.0 & -0.6 \\
\hline 2FGL J0211.2+1050 & 7.4 & 43 & 1.5 & 3.6 & 0.48 & 18 & 518 & 0.027 & 4.2 & 1.1 \\
\hline 2EG J0216+1107 & 7.4 & 43 & 1.2 & 4.7 & 0.48 & 15 & 543 & 0.038 & -5.7 & -1.3 \\
\hline 2FGL J0229.3-3644 & 6.1 & 14 & 1.8 & 2.0 & 0.39 & 7 & 421 & 0.021 & -1.8 & -0.6 \\
\hline RBS 334 & 6.1 & 14 & 0.5 & 5.6 & 0.35 & 26 & 293 & 0.083 & 1.6 & 0.3 \\
\hline RBS 0413 & 4.1 & 43 & 0.5 & 3.7 & 0.71 & 10 & 102 & 0.083 & 1.5 & 0.5 \\
\hline RBS 421 & 14.4 & 9 & 0.5 & 13.3 & 0.29 & 92 & 1153 & 0.083 & -4.1 & -0.4 \\
\hline 1ES $0323+022$ & 10.0 & 27 & 0.5 & 9.3 & 0.26 & 78 & 985 & 0.083 & -4.1 & -0.4 \\
\hline QSO B0331-362 & 30.6 & 19 & 1.1 & 20.6 & 0.24 & 109 & 3166 & 0.038 & -12.6 & -1.1 \\
\hline 2FGL J0334.3-3728 & 24.7 & 18 & 1.6 & 11.4 & 0.26 & 84 & 2656 & 0.025 & 16.6 & 1.9 \\
\hline PKS 0352-686 & 15.0 & 47 & 0.5 & 14.2 & 0.71 & 36 & 423 & 0.083 & 0.8 & 0.1 \\
\hline 2FGL J0426.6+0509c & 11.9 & 30 & 1.7 & 5.1 & 0.29 & 47 & 2137 & 0.023 & -1.8 & -0.3 \\
\hline $3 \mathrm{C} 120$ & 11.9 & 30 & 0.5 & 11.1 & 0.29 & 108 & 1008 & 0.083 & 24.0 & 2.4 \\
\hline 2FGL J0505.8-0411 & 8.3 & 21 & 1.1 & 5.8 & 0.29 & 54 & 1306 & 0.035 & 7.9 & 1.1 \\
\hline 1FGL J0506.9-5435 & 2.1 & 32 & 0.5 & 2.0 & 0.95 & 2 & 41 & 0.083 & -1.4 & -0.8 \\
\hline 1ES 0507-040 & 8.3 & 21 & 0.5 & 7.7 & 0.32 & 52 & 614 & 0.083 & 0.8 & 0.1 \\
\hline 2FGL J0515.0-4411 & 20.9 & 29 & 1.8 & 7.4 & 0.24 & 61 & 2877 & 0.021 & 0.1 & 0.0 \\
\hline 2FGL J0516.5-4601 & 20.9 & 29 & 0.8 & 17.1 & 0.26 & 132 & 2123 & 0.056 & 12.8 & 1.1 \\
\hline Pictor A & 20.9 & 29 & 0.5 & 19.4 & 0.29 & 134 & 1367 & 0.083 & 20.1 & 1.8 \\
\hline 2FGL J0537.7-5716 & 8.8 & 33 & 2.0 & 2.7 & 0.35 & 19 & 1103 & 0.019 & -1.8 & -0.4 \\
\hline 2FGL J0540.4-5415 & 8.8 & 33 & 1.5 & 4.7 & 0.35 & 26 & 1303 & 0.027 & -8.9 & -1.6 \\
\hline BZB J0543-5532 & 8.8 & 33 & 0.5 & 8.1 & 0.39 & 49 & 652 & 0.083 & -5.3 & -0.7 \\
\hline 1ES 0715-259 & 5.7 & 13 & 1.9 & 1.9 & 0.32 & 15 & 788 & 0.021 & -1.4 & -0.4 \\
\hline RBS 1049 & 4.3 & 30 & 0.5 & 3.9 & 0.39 & 17 & 253 & 0.083 & -4.1 & -0.9 \\
\hline 1ES $1218+30.4$ & 2.3 & 56 & 0.5 & 2.1 & 1.41 & 12 & 85 & 0.083 & 4.9 & 1.6 \\
\hline 2FGL J1226.0+2953 & 2.3 & 56 & 1.2 & 1.4 & 1.41 & 10 & 147 & 0.031 & 5.4 & 2.1 \\
\hline $3 \mathrm{C} 279$ & 5.5 & 26 & 0.5 & 5.0 & 0.29 & 35 & 475 & 0.075 & -0.5 & -0.1 \\
\hline 1ES 1332-295 & 10.1 & 25 & 0.7 & 8.4 & 0.26 & 54 & 1059 & 0.054 & -2.9 & -0.4 \\
\hline PKS $1345+125$ & 7.9 & 37 & 0.7 & 6.7 & 0.53 & 22 & 351 & 0.056 & 2.5 & 0.5 \\
\hline 2FGL J1351.4+1115 & 7.9 & 37 & 1.6 & 3.6 & 0.48 & 7 & 531 & 0.026 & -6.6 & -2.0 \\
\hline 1ES $1440+122$ & 11.2 & 37 & 0.5 & 10.4 & 0.29 & 66 & 650 & 0.083 & 11.8 & 1.5 \\
\hline 2FGL J1959.1-4245 & 12.9 & 33 & 2.1 & 2.7 & 0.39 & 8 & 994 & 0.016 & -8.1 & -2.2 \\
\hline PKS 2004-447 & 25.6 & 33 & 0.5 & 23.5 & 0.39 & 110 & 1139 & 0.083 & 15.1 & 1.4 \\
\hline RBS 1752 & 25.1 & 16 & 0.5 & 23.1 & 0.29 & 149 & 2023 & 0.083 & -19.6 & -1.5 \\
\hline PG 2209+184 & 8.8 & 42 & 0.5 & 8.1 & 0.64 & 19 & 286 & 0.083 & -4.8 & -1.0 \\
\hline 2FGL J2219.1+1805 & 8.8 & 42 & 1.9 & 2.6 & 0.64 & 7 & 529 & 0.019 & -3.2 & -1.1 \\
\hline RBS 1888 & 7.9 & 14 & 0.5 & 7.3 & 0.22 & 74 & 916 & 0.077 & 3.5 & 0.4 \\
\hline 3EG J2248+1745 & 17.3 & 43 & 1.8 & 5.8 & 0.48 & 36 & 1069 & 0.024 & 10.0 & 1.8 \\
\hline NGC 7469 & 7.9 & 33 & 0.5 & 7.4 & 0.32 & 79 & 772 & 0.083 & 14.7 & 1.7 \\
\hline PMN J2345-1555 & 21.0 & 15 & 1.0 & 15.9 & 0.22 & 147 & 3775 & 0.037 & 6.4 & 0.5 \\
\hline 1ES 2343-151 & 21.0 & 15 & 0.7 & 18.4 & 0.22 & 156 & 2629 & 0.066 & -18.5 & -1.4 \\
\hline 2FGL J2347.9-1629 & 21.0 & 15 & 1.6 & 9.6 & 0.20 & 104 & 3593 & 0.025 & 15.1 & 1.5 \\
\hline
\end{tabular}

Notes. The first five columns give the characteristics of the observation (target name, duration, zenith angle, average wobble offset and acceptancecorrected time). Column 6 is the energy threshold. The number of ON and OFF events above the energy threshold, and the normalization of the OFF events, $\alpha$, are shown in Cols. 7-9. The resulting excess and significance are given in the last two columns.

scale. A search on shorter time scales is ruled out by the small statistics in each temporal bin, and larger bins would result, for some of the targets, in a number of points that is too small to lead such a study.

\section{Discussion}

Among the 47 candidates, four blazars have been detected by other IACTs. The BL Lac object RBS 0413 has been discovered by the VERITAS Collaboration (Aliu et al. 2012) at the 1\% C.U. level, in agreement with the upper limit of $2.2 \%$ C.U. set in this study. 1ES 1218+30.4, detected by the MAGIC (Albert et al. 2006) and VERITAS (Acciari et al. 2009, 2010) Collaborations, is a known variable BL Lac object, with reported fluxes between $6 \%$ and $20 \%$ C.U. These are on the order of the upper limit of $8.0 \%$ measured above the comparably high energy threshold of 1.4 TeV. The VHE flux of the FSRQ 3C 279 has been measured at the $0.5 \%$ C.U. level by the MAGIC Collaboration (Aleksić et al. 2011) and is compatible with the 1.2\% C.U. derived here. The last detected $\mathrm{BL}$ Lac object in the list of targets is $1 \mathrm{ES} 1440+122$, with a flux of $1 \%$ C.U. (Benbow et al. 2011) that matches the upper limit derived in this paper.

The upper limits on 3C 120 and NGC 7469 are a factor of two higher than those derived in HUL1, despite a doubled amount of data. This can be related to background fluctuations, 
Table 4. Spectral and temporal analysis of 47 AGN.

\begin{tabular}{|c|c|c|c|c|c|}
\hline Object & $\begin{array}{c}E_{\mathrm{th}} \\
{[\mathrm{TeV}]}\end{array}$ & $\begin{array}{c}I\left(>E_{\mathrm{th}}\right) \\
{\left[\times 10^{-12} \mathrm{~cm}^{-2} \mathrm{~s}^{-1}\right]}\end{array}$ & $\begin{array}{l}I\left(>E_{\mathrm{th}}\right) \\
{[\% \text { C.U. }]}\end{array}$ & $\begin{array}{c}\mathcal{P}\left(\chi^{2}\right) \\
{[\%]}\end{array}$ & MJD-50 000 \\
\hline IIIZw 2 & 0.39 & 0.67 & $0.7^{U}$ & 22 & $\begin{array}{l}3943-44,3953,4267,4270,4272,4274-76,4279,4320, \\
4322-26,4328,4331-33\end{array}$ \\
\hline 1FGL J0022.2-1850 & 0.24 & 0.85 & $0.4^{U}$ & 79 & $\begin{array}{l}4653-60,5064,5090-92,5094,5112,5115-18,5415 \\
5417,5419,5422-27,5443-44,5448-51,5482,5501, \\
5504,5506-08,5775-76,5783,5885,5887-91,5910,5912\end{array}$ \\
\hline 2FGL J0048.8-6347 & 0.58 & 1.18 & $2.3^{U}$ & 50 & $5833-37$ \\
\hline PKS 0048-097 & 0.26 & 0.88 & $0.5^{U}$ & 69 & $\begin{array}{l}4023,4050-57,4321-26,4328,4331-35,4349,4350 \\
4352-53,4357,4359-60,4363,4374,4378-79,4381-85 \\
5058,5060,5063-65,5067-68\end{array}$ \\
\hline 1FGL J0051.4-6242 & 0.58 & 0.47 & $0.9^{U}$ & 32 & $5833-37$ \\
\hline RGB J0109+182 & 0.71 & 0.67 & $1.8^{U}$ & 15 & 5093,5095 \\
\hline 2FGL J0211.2+1050 & 0.48 & 1.21 & $1.7^{U}$ & 39 & $3966-69,3971-72,3974,3976-78$ \\
\hline 2EG J0216+1107 & 0.48 & 0.66 & $0.9^{U}$ & 63 & $3966-67,3969,3971-72,3974,3976-78$ \\
\hline 2FGL J0229.3-3644 & 0.39 & 1.05 & $1.1^{U}$ & 67 & $5444,5446,5448-52$ \\
\hline RBS 334 & 0.35 & 1.47 & $1.3^{U}$ & 31 & $5444,5446,5448-52$ \\
\hline RBS 0413 & 0.71 & 0.80 & $2.2^{D}$ & 22 & $5446,5448-51,5482-83$ \\
\hline RBS 421 & 0.29 & 0.89 & $0.6^{U}$ & 98 & $4715,4717,4720,4815,4818-20,4822-30$ \\
\hline $1 \mathrm{ES} 0323+022$ & 0.26 & 1.29 & $0.7^{U}$ & 84 & $3267-68,3675-77,3996-4000$ \\
\hline QSO B0331-362 & 0.24 & 0.82 & $0.4^{U}$ & 89 & $\begin{array}{l}3590,3592,3594-95,3597-98,3623,3625-27, \\
3638-39,3641-42,3643-44,4353,4358,4360-61, \\
4363-64,4379-86,4391\end{array}$ \\
\hline 2FGL J0334.3-3728 & 0.26 & 1.77 & $0.9^{U}$ & 14 & $\begin{array}{l}3589-90,3592,3597-98,3623,3625-27,3637-38,3641-44, \\
4353,4358,4360-61,4363-64,4378-86,4391\end{array}$ \\
\hline PKS 0352-686 & 0.71 & 0.40 & $1.1^{U}$ & 43 & $\begin{array}{l}5483-84,5499-5502,5504-08,5510-12,5526-27,5529, \\
5532-37\end{array}$ \\
\hline 2FGL J0426.6+0509c & 0.29 & 1.57 & $1.0^{U}$ & 30 & $3315-17,3352-54,5834-39,5841-43,5867-68$ \\
\hline $3 \mathrm{C} 120$ & 0.29 & 2.23 & 1.4 & 73 & $3315-18,3352-54,5834-43,5867-68$ \\
\hline 2FGL J0505.8-0411 & 0.29 & 2.14 & $1.3^{U}$ & 45 & $4439,4441-46,4450$ \\
\hline 1FGL J0506.9-5435 & 0.95 & 0.52 & $2.3^{U}$ & 87 & $5867-68$ \\
\hline 1ES 0507-040 & 0.32 & 1.37 & $1.0^{U}$ & 69 & $4439,4441-46,4450$ \\
\hline 2FGL J0515.0-4411 & 0.24 & 1.94 & $0.9^{U}$ & 27 & $\begin{array}{l}3268-70,3273,3318-19,3350,3352-53,4050-53 \\
4055-56,4059-62,4496,4498-99,4819-20,4823\end{array}$ \\
\hline 2FGL J0516.5-4601 & 0.26 & 1.63 & $0.9^{U}$ & 89 & $\begin{array}{l}3268-70,3273,3318-19,3350,3352-53,4051-53 \\
4055-56,4059-62,4496,4499,4819-20,4823\end{array}$ \\
\hline Pictor A & 0.29 & 1.44 & $0.9^{U}$ & 12 & $\begin{array}{l}3268-70,3273,3318-19,3350,3352-53,4050-53 \\
4055-56,4059-62,4496,4498-99,4819-20,4823\end{array}$ \\
\hline 2FGL J0537.7-5716 & 0.35 & 2.03 & $1.7^{U}$ & 78 & $5911,5914,5917,5922-25$ \\
\hline 2FGL J0540.4-5415 & 0.35 & 1.16 & $1.0^{U}$ & 27 & $5911,5914,5917,5922-25$ \\
\hline BZB J0543-5532 & 0.39 & 0.90 & $0.9^{U}$ & 25 & $5911,5914,5917,5922-25$ \\
\hline 1ES 0715-259 & 0.32 & 2.09 & $1.5^{U}$ & 96 & $4140-44,4146,4148$ \\
\hline RBS 1049 & 0.39 & 1.16 & $1.2^{U}$ & 22 & $5320-23$ \\
\hline 1ES $1218+30.4$ & 1.41 & 0.97 & $8.0^{D}$ & 19 & $3875-76$ \\
\hline 2FGL J1226.0+2953 & 1.41 & 1.31 & $11^{U}$ & 90 & $3875-76$ \\
\hline 3C 279 & 0.29 & 1.85 & $1.2^{D}$ & 40 & $4118-21,4501,4855,4858-59,4861$ \\
\hline 1ES 1332-295 & 0.26 & 1.53 & $0.8^{U}$ & 45 & $3929-35$ \\
\hline PKS $1345+125$ & 0.53 & 0.68 & $1.1^{U}$ & 21 & $4938-41,4944-46,4948-49,4952$ \\
\hline 2FGL J1351.4+1115 & 0.48 & 0.51 & $0.7^{U}$ & 40 & $4938-41,4944-46,4948-49,4952$ \\
\hline 1ES $1440+122$ & 0.29 & 1.66 & $1.0^{D}$ & 47 & $3109,3119,4995-99,5002-03,5005-06$ \\
\hline 2FGL J1959.1-4245 & 0.39 & 1.01 & $1.0^{U}$ & 95 & $\begin{array}{l}5358-59,5362,5365,5367,5369,5386,5389-91,5393-94, \\
5396-97,5413,5415-16,5419,5421-23\end{array}$ \\
\hline PKS 2004-447 & 0.39 & 0.88 & $0.9^{U}$ & 26 & $\begin{array}{l}5358-59,5361-62,5364-67,5369-70,5386-87,5389-90 \\
5391-96,5413-16,5418-24\end{array}$ \\
\hline RBS 1752 & 0.29 & 0.56 & $0.3^{U}$ & 37 & $4625-32,4653-56,4728-39$ \\
\hline PG 2209+184 & 0.64 & 0.38 & $0.9^{U}$ & 52 & $4373,4375-76,4378-79,4381-86$ \\
\hline 2FGL J2219.1+1805 & 0.64 & 0.42 & $1.0^{U}$ & 47 & $4374,4376-79,4381-86$ \\
\hline RBS 1888 & 0.22 & 2.16 & $0.9^{U}$ & 94 & $3207-10,3914-18$ \\
\hline 3EG J2248+1745 & 0.48 & 1.10 & $1.6^{U}$ & 99 & 4292-96,4298-04,5004-09 \\
\hline NGC 7469 & 0.32 & 1.80 & 1.3 & 70 & $3202,3206,3211-12,4019-20,4022-23$ \\
\hline PMN J2345-1555 & 0.22 & 1.65 & $0.7^{U}$ & 47 & $3211-13,3590,3592-95,3597-98,5495-96,5498-99$ \\
\hline 1ES 2343-151 & 0.22 & 0.97 & $0.4^{U}$ & 20 & $\begin{array}{l}3212-13,3590,3592-93,3594-95,3597-98,5495-96, \\
5498-99\end{array}$ \\
\hline 2FGL J2347.9-1629 & 0.20 & 3.15 & $1.1^{U}$ & 88 & $\begin{array}{l}3211-12,3590,3592-93,3594-95,3597-98,5495-96 \\
5498-99\end{array}$ \\
\hline
\end{tabular}

Notes. The upper limits given in Cols. 3 and 4 are computed at the $99.9 \%$ level. The superscript ${ }^{U}$ indicates the best VHE upper limit computed for this target to date and ${ }^{D}$ corresponds to a source detected by another VHE instrument. The observation nights are listed in the last column, and the $\chi^{2}$ probabilities for a constant fit of the flux at this time scale are shown in Col. 5. 
H.E.S.S. Collaboration: Flux upper limits for 47 AGN

Table 5. Comparison of the high-energy extrapolation from the 2LAC with H.E.S.S. upper limits.

\begin{tabular}{lccccl}
\hline \hline Object & $z$ & $\begin{array}{c}E_{\mathrm{th}} \\
{[\mathrm{TeV}]}\end{array}$ & $\begin{array}{c}I\left(>E_{\mathrm{th}}\right) \\
{[\% \text { C.U.] }}\end{array}$ & $\begin{array}{c}I_{2 \mathrm{LAC}}\left(>E_{\mathrm{th}}\right) \\
{[\% \text { C.U.] }}\end{array}$ & $\begin{array}{c}I_{2 \mathrm{LAC}}^{\mathrm{EBL}}\left(>E_{\mathrm{th}}\right) \\
{[\% \text { C.U.] }}\end{array}$ \\
\hline 2FGL J1351.4+1115 & 0.40 & 0.48 & 0.7 & 40 & 0.2 \\
1FGL J0022.2-1850 & $0.77-1.38$ & 0.24 & 0.4 & 24 & 0.1 \\
1FGL J0051.4-6242 & $<1.12$ & 0.58 & 0.9 & 37 & 0.5 \\
BZB J0543-5532 & 0.27 & 0.39 & 0.9 & 25 & 1.4 \\
1FGL J0506.9-5435 & $<1.07$ & 0.95 & 2.3 & 65 & 0.2 \\
RBS 334 & 0.41 & 0.35 & 1.3 & 13 & 0.2 \\
PKS 0048-097 & 0.64 & 0.26 & 0.5 & 3.6 & 0.05 \\
2FGL J0334.3-3728 & $<1.34$ & 0.26 & 0.9 & 7.3 & 1.0 \\
RBS 1049 & 0.25 & 0.39 & 1.2 & 5.0 & 0.4 \\
PMN J2345-1555 & 0.62 & 0.22 & 0.7 & 2.7 & 0.1 \\
RBS 421 & 0.29 & 0.29 & 0.6 & 1.8 & 0.2 \\
RBS 1752 & 0.45 & 0.29 & 0.3 & 1.0 & 0.04 \\
\hline
\end{tabular}

Notes. Only objects with constraining limits are selected. $I_{2 \mathrm{LAC}}\left(>E_{\mathrm{th}}\right)$ and $I_{2 \mathrm{LAC}}^{\mathrm{EBL}}\left(>E_{\mathrm{th}}\right)$ are the $2 \mathrm{LAC}$ measurements extrapolated above $E_{\mathrm{th}}$, taking into account the EBL absorption for the second quantity. When only an upper limit on the redshift is available, a value of $z=0.3$ is assumed to derive these extrapolations. For 1FGL J0022.2-1850, the lower limit $z>0.77$ is used.
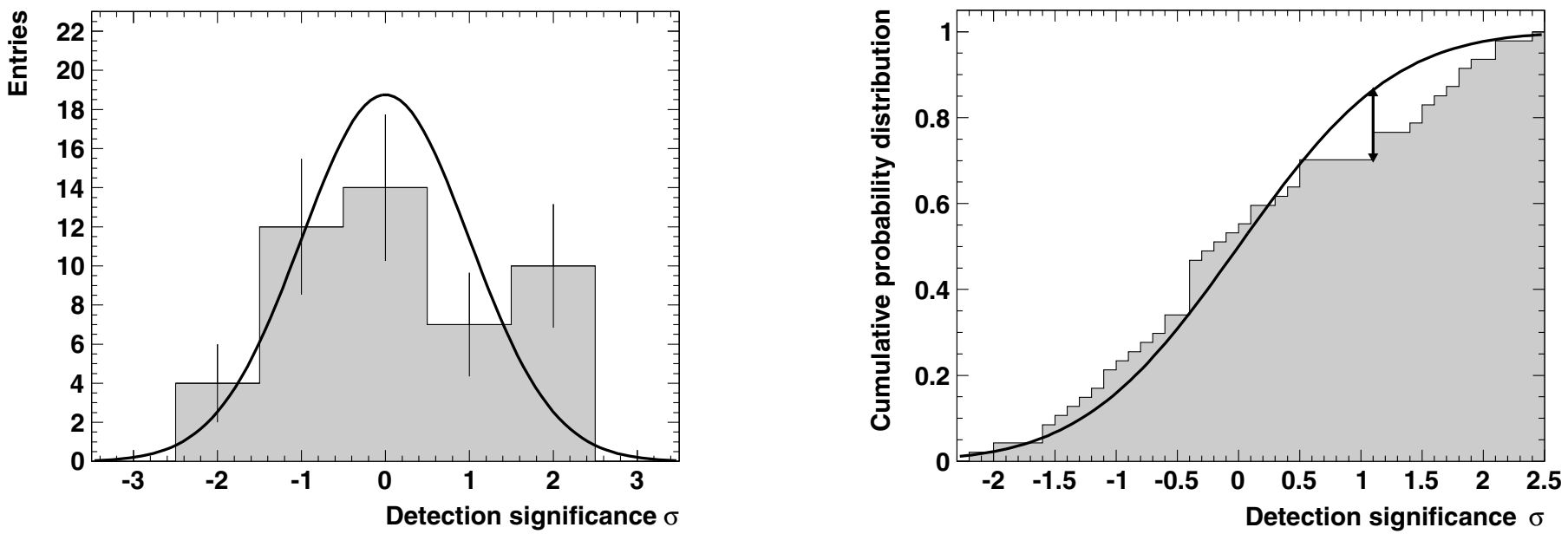

Fig. 1. Left: distribution of the detection significances, $\sigma$, for the 47 candidates using $1 \sigma$-wide bins. Error bars indicate the square root of the number of events in each bin, and the black line is a normal distribution of 47 events centered on zero and of unit width. Right: cumulative distribution function of the detection significance and of the normal distribution. The maximum distance between the distributions is shown as a double-headed arrow.
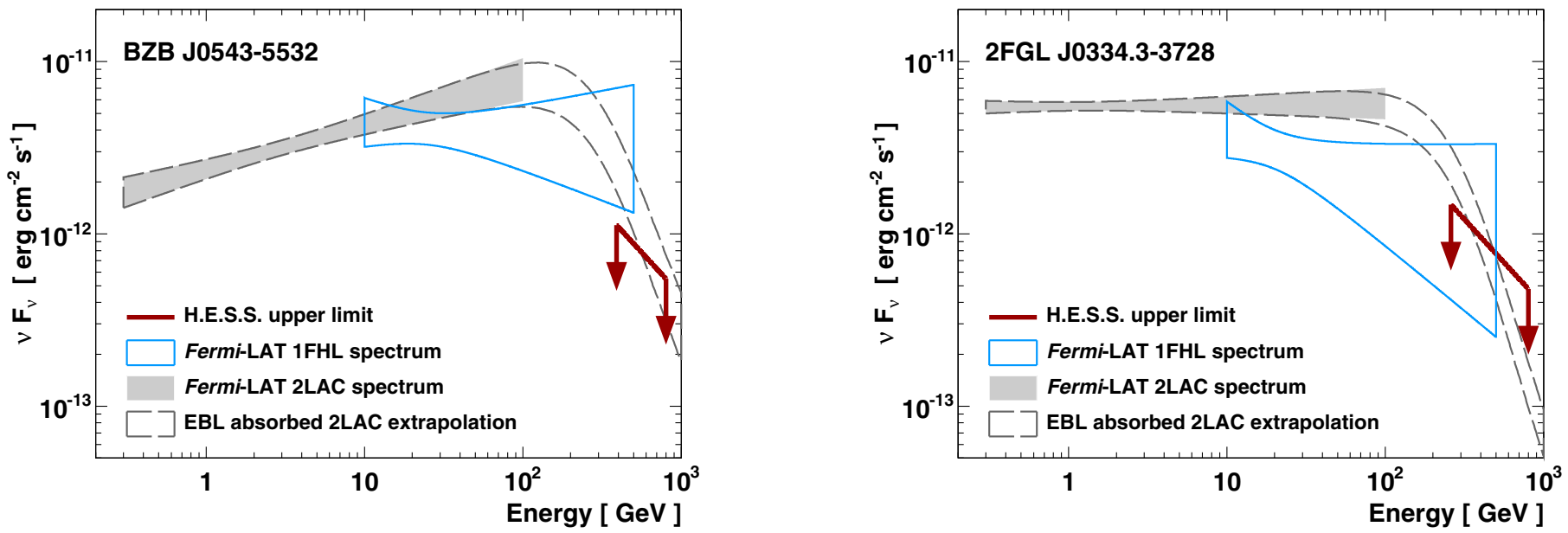

Fig. 2. Left: HE $\gamma$-ray spectrum and VHE upper limit on the emission of BZB J0543-5532 as measured by Fermi-LAT and H.E.S.S. The EBLabsorbed HE extrapolation based on the 2LAC is shown with a dashed line. Right: HE $\gamma$-ray spectrum and VHE upper limit on the emission of 2FGL J0334.3-3728. For this object, a fiducial redshift of 0.3 is assumed in the extrapolation. 
with negative detection significances of $\sim-2 \sigma$ in HUL1 and $\sim+2 \sigma$ upward fluctuations observed in this study.

For the other targets, that is 41 among the $47 \mathrm{AGN}$, the upper limits derived in Table 4 are the strongest reported to date ${ }^{5}$, with values down to $0.4 \%$ C.U. These upper limits are compared with the HE flux reported in the 2LAC, extrapolated above the threshold energy of H.E.S.S., $I_{2 \mathrm{LAC}}\left(>E_{\mathrm{th}}\right)$, without taking into account absorption by the EBL. Since H.E.S.S. observed the sky for a longer period than Fermi-LAT, the 2LAC spectra are not strictly simultaneous with the VHE constraints. The comparison of the Fermi-LAT extrapolated fluxes and of the H.E.S.S. upper limits is thus based on the assumption that the 2LAC spectra are representative of the average HE emission. This assumption is corroborated by $2 \mathrm{LAC}$ studies of FSRQs and BL Lac objects that show an average fractional variance of the flux (square root of the normalized excess variance) on the order of $0.55 \pm 0.10$, that is fluxes that vary on average within $\pm 55 \%$, and also by a rather short duty cycle for high flux events (above 1.5 standard deviation), with a most probable value for the duty cycle on the order of $5 \%$ to $10 \%$. The targets for which $I_{2 \mathrm{LAC}}\left(>E_{\mathrm{th}}\right)$ is at least twice as high ${ }^{6}$ as the H.E.S.S. upper limit are listed in Table 5. Sources detected with other IACTs as well as the distant 2FGL J0537.7$5716(z=1.55)$ are not included in the list.

The extrapolated fluxes of these sources are higher than the H.E.S.S. upper limits, indicating curvature in their spectra. The curvature can have an intrinsic (i.e. related to the underlying emitting particles) and extrinsic (i.e. due to the EBL absorption) origin. To constrain the origin of this curvature, the Fermi-LAT fluxes, $\phi_{2 \mathrm{LAC}}(E)$, were extrapolated taking into account the best-fit EBL model derived with the H.E.S.S. data, corresponding to the optical depth of Franceschini et al. (2008), $\tau_{\mathrm{FR} 08}(E, z)$ scaled up by a factor $\alpha_{0}=1.27$ (Abramowski et al. 2013d). The EBL-absorbed extrapolations are thus computed as $I_{2 \mathrm{LAC}}^{\mathrm{EBL}}\left(>E_{\text {th }}\right)=\int_{E_{\text {th }}} \mathrm{d} E \phi_{2 \mathrm{LAC}}(E) \mathrm{e}^{-\alpha_{0} \times \tau_{\mathrm{FR} 08}(E, z)}$. Targets for which only an upper limit on the redshift was available were assumed to lie at $z=0.3$, roughly corresponding to the peak of the distribution for BL Lac objects in the 2LAC. For 1FGL J0022.2-1850, the EBL-absorbed extrapolation derived using a redshift of 0.77 does not exceed the H.E.S.S. upper limit.

When taking into account the EBL absorption, all but two of the HE extrapolations lie below the H.E.S.S. upper limits, indicating that no intrinsic curvature is required to explain the observed spectral break. BZB J0543-5532 is an exception, with a VHE upper limit a factor of two lower than the EBL-absorbed extrapolation. A straight power-law extrapolation of the intrinsic emission is therefore rejected, suggesting an intrinsic break in the photon spectrum. This curvature is also suggested by the marginal agreement between the H.E.S.S. upper-limit and the high-energy end of the spectrum from the Fermi-LAT Catalog of Sources Above $10 \mathrm{GeV}$ (1FHL, The Fermi-LAT Collaboration 2013). Similar conclusions can be drawn for 2FGL J0334.33728 , though with smaller statistics from the 1FHL and under the assumption that the object is nearby $(z<0.3)$. Tighter constraints on the distance of this source and an increased coverage with Fermi-LAT and H.E.S.S. will allow for more definite conclusions on the intrinsic emission of the source.

\footnotetext{
5 Variations in the energy thresholds of different instruments that observed the same targets were taken into account when comparing upper limits. Values are also reported in Crab units in this paper for the sake of clarity.

6 A fiducial value of two corresponds to the average EBL absorption between $500 \mathrm{GeV}$ and $1 \mathrm{TeV}$ for a source situated at $z \sim 0.1$ (e.g., within the modeling of Franceschini et al. 2008).
}

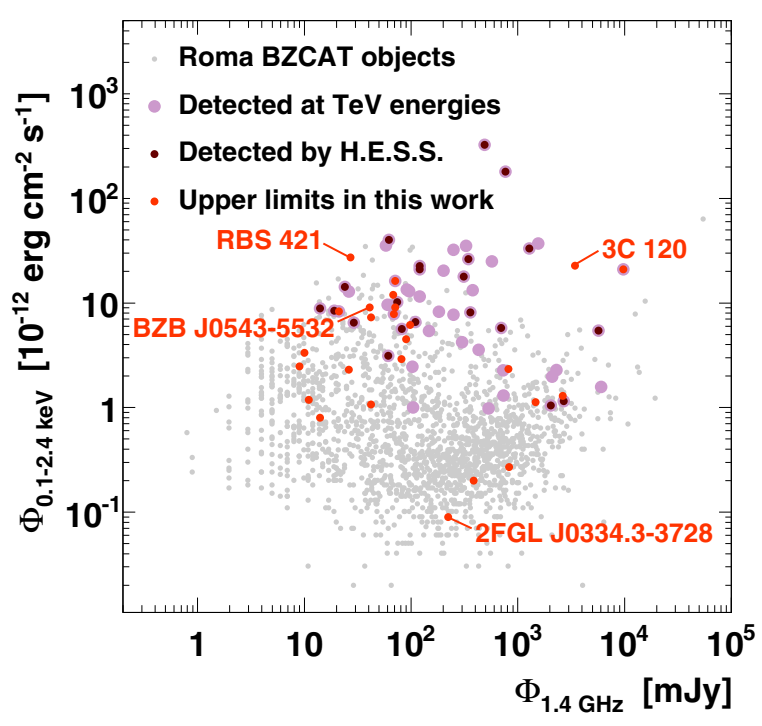

Fig. 3. X-ray flux in the $0.1-2.4 \mathrm{keV}$ band vs radio flux at $1.4 \mathrm{GHz}$ for objects listed in the Roma BZCAT Catalog. 50 of the 56 AGN detected at VHE (as of the end 2013) are listed in the BZCAT with detected X-ray (ROSAT) and radio (NVSS/SUMSS) emission. 25 of the 47 objects studied in this paper are shown, with a selection biased toward X-ray-bright BL Lac objects. Based on ROMA BZCAT and TeVCat.

With the launch of Fermi-LAT, the AGN observation strategy at VHE has partly shifted from a target selection based on radio and X-ray fluxes towards a selection based on extrapolations of HE spectra. It should be noted nonetheless that, based only on the latter criterion, a fourth of the sources listed in Table 1 would not have been discovered. High-frequency-peaked BL Lac objects such as PKS 0548-322, SHBL J001355.9185406, 1ES 1312-423, 1ES 0229+200, and 1ES 0347-121 are indeed not listed in the 2LAC because of a hard but faint HE emission.

Broadband multiwavelength strategies prove to be of critical importance in such cases. As discussed in Costamante \& Ghisellini (2002) and illustrated in Fig. 3, bright TeV BL Lac objects tend to have bright X-ray and radio counterparts. The latter criterion is not sufficient, however, as it tends to discard FSRQs with their low X-ray fluxes (low-energy component peaking in the optical-infrared band) and as X-ray bright objects, such as RBS 421 or 3C 120 studied in this paper, do not necessarily show bright $\mathrm{TeV}$ counterparts. Good HE-based candidates do not necessarily cluster in the upper-right corner of Fig. 3 either, as shown by the relatively low radio flux of BZB J0543-5532 and the low X-ray flux of 2FGL J0334.3-3728.

The extension of the population of AGN detected at VHE and the discovery of new types of sources will be a primary task of the future Cherenkov Telescope Array, CTA (Sol et al. 2013; Reimer \& Böttcher 2013). A best-suited target selection will account both for multiwavelength information from radio, X-ray, optical, and HE instruments, and for the charting effort led by previous and current-generation VHE instruments.

\section{Conclusion}

A large sample of AGN has been observed with H.E.S.S. since 2002, resulting in the discovery of more than a third of the known extragalactic VHE emitters. Observations of 47 targets without significant excess were selected and upper limits on their integral fluxes were computed. For 41 of these objects, the upper limits derived in this paper are the strongest to date.

No significant flaring event was detected during the $\sim 400 \mathrm{~h}$ of observation of the 47 targets. The straight extrapolation of the 
HE emission is challenged by the VHE upper limit for a dozen objects. For all but two of them, this spectral curvature can be accounted for by the interaction of $\gamma$ rays with the EBL.

Active galactic nuclei observations, which are crucial both for the understanding of the EBL and of the objects themselves, will remain a primary goal of H.E.S.S. during its second phase, H.E.S.S. II, where observations at lower energies will increase the number of detected sources and the maximum redshift accessible by Cherenkov telescopes. Extensive campaigns probing the sky down to fractions of percent of the Crab Nebula flux remain a major task of current VHE telescopes. This tremendous effort is paving the way for targeted AGN observations with CTA.

Acknowledgements. The support of the Namibian authorities and of the University of Namibia in facilitating the construction and operation of H.E.S.S. is gratefully acknowledged, as is the support of the German Ministry for Education and Research (BMBF), the Max Planck Society, the German Research Foundation (DFG), the French Ministry for Research, the CNRS-IN2P3 and the Astroparticle Interdisciplinary Programme of the CNRS, the UK Science and Technology Facilities Council (STFC), the IPNP of the Charles University, the Czech Science Foundation, the Polish Ministry of Science and Higher Education, the South African Department of Science and Technology and National Research Foundation, and by the University of Namibia. We appreciate the excellent work of the technical support staff in Berlin, Durham, Hamburg, Heidelberg, Palaiseau, Paris, Saclay, and in Namibia in the construction and operation of the equipment. This research has made use of the SIMBAD database, operated at the CDS, Strasbourg, France.

\section{References}

Abramowski, A., Acero, F., Aharonian, F., et al. 2010 (H.E.S.S. Collaboration), A\&A, 516, A56

Abramowski, A., Acero, F., Aharonian, F., et al. (H.E.S.S. Collaboration) 2011a, A\&A, 529, A49

Abramowski, A., Acero, F., et al. (H.E.S.S. Collaboration) 2011b, A\&A, 533, A110

Abramowski, A., Acero, F., Aharonian, F., et al. 2012a (H.E.S.S. Collaboration), ApJ, 746, 151

Abramowski, A., Acero, F., et al. (H.E.S.S. Collaboration) 2012b, A\&A, 538, A103

Abramowski, A., Acero, F., Aharonian, F., et al. 2012c (H.E.S.S. Collaboration), A\&A, 539, A149

Abramowski, A., Acero, F., et al. (H.E.S.S. Collaboration) 2012d, A\&A, 542, A94

Abramowski, A., Acero, F., et al. (H.E.S.S. Collaboration) 2013a, A\&A, 559, A136

Abramowski, A., Acero, F., et al. (H.E.S.S. Collaboration) 2013b, A\&A, 554, A72

Abramowski, A., Acero, F., et al. (H.E.S.S. Collaboration) 2013c, A\&A, 554, A107

Abramowski, A., Acero, F., et al. (H.E.S.S. Collaboration) 2013d, A\&A, 550, A4

Abramowski, A., Acero, F., et al. (H.E.S.S. Collaboration) 2013e, A\&A, 552, A118

Abramowski, A., Acero, F., et al. (H.E.S.S. Collaboration) 2013f, MNRAS, 434, 1889

Acciari, V. A., Aliu, E., Arlen, T., et al. 2009, ApJ, 695, 1370

Acciari, V. A., Aliu, E., Beilicke, M., et al. 2010, ApJ, 709, L163

Ackermann, M., Ajello, M., Allafort, A., et al. 2011, ApJ, 743, 171

Adelman-McCarthy, J. K., et al. 2008, VizieR Online Data Catalog: II/282

Adelman-McCarthy, J. K., et al. 2009, VizieR Online Data Catalog: II/294

Aharonian, F., Akhperjanian, A., Beilicke, M., et al. 2004, A\&A, 421, 529

Aharonian, F., Akhperjanian, A. G., Aye, K.-M., et al. 2005a (H.E.S.S. Collaboration), A\&A, 437, 95

Aharonian, F., Akhperjanian, A. G., Bazer-Bachi, A. R., et al. 2005 b (H.E.S.S. Collaboration), A\&A, 441, 465

Aharonian, F., Akhperjanian, A. G., Bazer-Bachi, A. R., et al. 2006a (H.E.S.S. Collaboration), A\&A, 457, 899

Aharonian, F., Akhperjanian, A. G., Bazer-Bachi, A. R., et al. 2006 b (H.E.S.S. Collaboration), Nature, 440, 1018

Aharonian, F., Akhperjanian, A. G., Barres de Almeida, U., et al. 2007a (H.E.S.S. Collaboration), A\&A, 475, L9

Aharonian, F., Akhperjanian, A. G., Barres de Almeida, U., et al. 2007b (H.E.S.S. Collaboration), A\&A, 473, L25
Aharonian, F., Akhperjanian, A. G., Bazer-Bachi, A. R., et al. 2007c (H.E.S.S. Collaboration), A\&A, 470, 475

Aharonian, F., Akhperjanian, A. G., Barres de Almeida, U., et al. 2008a (H.E.S.S. Collaboration), A\&A, 477, 481

Aharonian, F., Akhperjanian, A. G., Barres de Almeida, U., et al. 2008b (H.E.S.S. Collaboration), A\&A, 478, 387

Aharonian, F., Akhperjanian, A. G., Barres de Almeida, U., et al. 2008c (H.E.S.S. Collaboration), A\&A, 481, L103

Aharonian, F., Akhperjanian, A. G., Anton, G., et al. 2009 (H.E.S.S Collaboration), ApJ, 695, L40

Aharonian, F., Akhperjanian, A. G., Anton, G., et al. 2010 (H.E.S.S Collaboration) A\&A, 521, A69

Albert, J., Aliu, E., Anderhub, H., et al. 2006, ApJ, 642, L119

Aleksić, J., Antonelli, L. A., Antoranz, P., et al. 2011, A\&A, 530, A4

Aliu, E., Archambault, S., Arlen, T., et al. 2012, ApJ, 750, 94

Atwood, W. B., Abdo, A. A., Ackermann, M., et al. 2009, ApJ, 697, 1071

Barkhouse, W. A., \& Hall, P. B. 2001, AJ, 121, 2843

Bauer, F. E., Condon, J. J., Thuan, T. X., \& Broderick, J. J. 2000, ApJS, 129, 547

Becherini, Y., Boisson, C., Cerruti, M., et al. 2012 (H.E.S.S. Collaboration), AIP Conf. Proc., 1505, 490

Beckmann, V., Gehrels, N., Shrader, C. R., \& Soldi, S. 2006, ApJ, 638, 642

Benbow, W., et al. 2011 [arXiv: 1110.0040 ]

Berge, D., Funk, S., \& Hinton, J. 2007, A\&A, 466, 1219

Carangelo, N., Falomo, R., Kotilainen, J., Treves, A., \& Ulrich, M.-H. 2003, A\&A, 412, 651

Costamante, L., \& Ghisellini, G. 2002, A\&A, 384, 56

de Naurois, M., \& Rolland, L. 2009, Astropart. Phys., 32, 231

Donato, D., Ghisellini, G., Tagliaferri, G., \& Fossati, G. 2001, A\&A, 375, 739

Dubus, G., Contreras, J. L., Funk, S., et al. 2013, Astropart. Phys., 43, 317

Falco, E. E., Kurtz, M. J., Geller, M. J., et al. 1999, PASP, 111, 438

Fischer, J.-U., Hasinger, G., Schwope, A. D., et al. 1998, Astron. Nachr., 319 347

Franceschini, A., Rodighiero, G., \& Vaccari, M. 2008, A\&A, 487, 837

Ghisellini, G., Tagliaferri, G., Foschini, L., et al. 2011, MNRAS, 411, 901

Giommi, P., Piranomonte, S., Perri, M., \& Padovani, P. 2005, A\&A, 434, 385

Healey, S. E., Romani, R. W., Cotter, G., et al. 2008, ApJS, 175, 97

Hernán-Caballero, A., \& Hatziminaoglou, E. 2011, MNRAS, 414, 500

Hofmann, W. (H.E.S.S. Collaboration) 2012, ATel, 4072, 1

Hook, I. M., Shaver, P. A., Jackson, C. A., Wall, J. V., \& Kellermann, K. I. 2003, A\&A, 399, 469

Horan, D., Badran, H. M., Bond, I. H., et al. 2004, ApJ, 603, 51

Jones, D. H., Read, M. A., Saunders, W., et al. 2009, MNRAS, 399, 683

Kovalev, Y. Y., Nizhelsky, N. A., Kovalev, Y. A., et al. 1999, A\&AS, 139, 545

Landt, H., Padovani, P., Perlman, E. S., \& Giommi, P. 2004, MNRAS, 351, 83

Laurent-Muehleisen, S. A., Kollgaard, R. I., Feigelson, E. D., Brinkmann, W., \& Siebert, J. 1999, ApJ, 525, 127

Lavaux, G., \& Hudson, M. J. 2011, MNRAS, 416, 2840

Li, T.-P., \& Ma, Y.-Q. 1983, ApJ, 272, 317

Liu, F. K., \& Zhang, Y. H. 2002, A\&A, 381, 757

Massaro, E., Giommi, P., Leto, C., et al. 2009, A\&A, 495, 691

Mazin, D., \& Raue, M. 2007, A\&A, 471, 439

Meisner, A. M., \& Romani, R. W. 2010, ApJ, 712, 14

Ohm, S., van Eldik, C., \& Egberts, K. 2009, Astropart. Phys., 31, 383

Pita, S., Goldoni, P., Boisson, C., et al. 2012, in AIP Conf. Ser. 1505, eds. F. A. Aharonian, W. Hofmann, \& F. M. Rieger, 566

Paturel, G., Dubois, P., Petit, C., \& Woelfel, F. 2002, LEDA, 0

Perlman, E. S. 2000, in AIP Conf. Ser. 515, eds. B. L. Dingus, M. H. Salamon, \& D. B. Kieda, 53

Rau, A., Schady, P., Greiner, J., et al. 2012, A\&A, 538, A26

Reimer, A., \& Böttcher, M. 2013, Astropart. Phys., 43, 103

Rodriguez, J., Tomsick, J. A., \& Chaty, S. 2009, A\&A, 494, 417

Rolke, W. A., López, A. M., \& Conrad, J. 2005, Nucl. Instrum. Methods Phys. Res. A, 551, 493

Sanchez, D., Giebels, B., Fortin, P., et al. 2012 (H.E.S.S. and Fermi-LAT Collaborations), in IAU Symp. 284, eds. R. J. Tuffs, \& C. C. Popescu, 411

Schachter, J. F., Stocke, J. T., Perlman, E., et al. 1993, ApJ, 412, 541

Schmidt, M. 1963, Nature, 197, 1040

Shaw, M. S., Romani, R. W., Cotter, G., et al. 2013, ApJ, 764, 135

Sol, H., Zech, A., Boisson, C., et al. 2013, Astropart. Phys., 43, 215

Sowards-Emmerd, D., Romani, R. W., Michelson, P. F., Healey, S. E., \& Nolan, P. L. 2005, ApJ, 626, 95

Stecker, F. W., de Jager, O. C., \& Salamon, M. H. 1996, ApJ, 473, L75

Tavecchio, F., Ghisellini, G., Ghirlanda, G., Foschini, L., \& Maraschi, L. 2010, MNRAS, 401, 1570

The Fermi-LAT Collaboration. 2013, ApJS, submitted [arXiv: 1306. 6772]

Urry, C. M., \& Padovani, P. 1995, PASP, 107, 803

Woo, J.-H., Urry, C. M., van der Marel, R. P., Lira, P., \& Maza, J. 2005, ApJ, 631,762 
1 Universität Hamburg, Institut für Experimentalphysik, Luruper Chaussee 149, 22761 Hamburg, Germany

2 Max-Planck-Institut für Kernphysik, PO Box 103980, 69029 Heidelberg, Germany

3 Dublin Institute for Advanced Studies, 31 Fitzwilliam Place, 2 Dublin, Ireland

${ }^{4}$ National Academy of Sciences of the Republic of Armenia, Yerevan, Armenia

5 Yerevan Physics Institute, 2 Alikhanian Brothers St., 375036 Yerevan, Armenia

6 Institut für Physik, Humboldt-Universität zu Berlin, Newtonstr. 15, 12489 Berlin, Germany

7 Universität Erlangen-Nürnberg, Physikalisches Institut, Erwin-Rommel-Str. 1, 91058 Erlangen, Germany

8 University of Durham, Department of Physics, South Road, Durham DH1 3LE, UK

9 DESY, 15738 Zeuthen, Germany

10 Institut für Physik und Astronomie, Universität Potsdam, KarlLiebknecht-Strasse 24/25, 14476 Potsdam, Germany

11 Nicolaus Copernicus Astronomical Center, ul. Bartycka 18, 00-716 Warsaw, Poland

12 Department of Physics and Electrical Engineering, Linnaeus University, 35195 Växjö, Sweden,

13 Institut für Theoretische Physik, Lehrstuhl IV: Weltraum und Astrophysik, Ruhr-Universität Bochum, 44780 Bochum, Germany

14 Institut für Astro- und Teilchenphysik, Leopold-FranzensUniversität Innsbruck, 6020 Innsbruck, Austria

15 Laboratoire Leprince-Ringuet, Ecole Polytechnique, CNRS/IN2P3, 91128 Palaiseau, France

16 now at Santa Cruz Institute for Particle Physics, Department of Physics, University of California at Santa Cruz, Santa Cruz CA 95064, USA

17 Centre for Space Research, North-West University, 2520 Potchefstroom, South Africa

18 LUTH, Observatoire de Paris, CNRS, Université Paris Diderot, 5 place Jules Janssen, 92190 Meudon, France

19 LPNHE, Université Pierre et Marie Curie Paris 6, Université Denis Diderot Paris 7, CNRS/IN2P3, 4 place Jussieu, 75252 Paris Cedex 5, France

20 Institut für Astronomie und Astrophysik, Universität Tübingen, Sand 1, 72076 Tübingen, Germany

21 DSM/Irfu, CEA Saclay, 91191 Gif-Sur-Yvette Cedex, France

22 Astronomical Observatory, The University of Warsaw, Al. Ujazdowskie 4, 00-478 Warsaw, Poland
23 Now at Harvard-Smithsonian Center for Astrophysics, 60 Garden Street, Cambridge MA 02138, USA

24 School of Physics, University of the Witwatersrand, 1 Jan Smuts avenue, Braamfontein, 2050 Johannesburg, South Africa

25 Landessternwarte, Universität Heidelberg, Königstuhl, 69117 Heidelberg, Germany

26 Oskar Klein Centre, Department of Physics, Stockholm University, Albanova University Center, 10691 Stockholm, Sweden

27 Wallenberg Academy Fellow

${ }^{28}$ Université Bordeaux 1, CNRS/IN2P3, Centre d'Études Nucléaires de Bordeaux-Gradignan, 33175 Gradignan, France

29 Funded by contract ERC-StG-259391 from the European Community

30 University of Namibia, Department of Physics, Private Bag 13301, Windhoek, Namibia

31 School of Chemistry \& Physics, University of Adelaide, 5005 Adelaide, Australia

32 APC, AstroParticule et Cosmologie, Université Paris Diderot, CNRS/IN2P3, CEA/Irfu, Observatoire de Paris, Sorbonne Paris Cité, 10, rue Alice Domon et Léonie Duquet, 75205 Paris Cedex 13, France,

33 UJF-Grenoble 1/CNRS-INSU, Institut de Planétologie et d'Astrophysique de Grenoble (IPAG) UMR 5274, 38041 Grenoble, France

34 Department of Physics and Astronomy, The University of Leicester, University Road, Leicester LE1 7RH, UK

35 Instytut Fizyki Jạdrowej PAN, ul. Radzikowskiego 152, 31-342 Kraków, Poland

${ }^{36}$ Laboratoire Univers et Particules de Montpellier, Université Montpellier 2, CNRS/IN2P3, CC 72, place Eugène Bataillon, 34095 Montpellier Cedex 5, France

37 Laboratoire d'Annecy-le-Vieux de Physique des Particules, Université de Savoie, CNRS/IN2P3, 74941 Annecy-le-Vieux, France

38 Obserwatorium Astronomiczne, Uniwersytet Jagielloński, ul. Orla 171, 30-244 Kraków, Poland

39 Toruń Centre for Astronomy, Nicolaus Copernicus University, ul. Gagarina 11, 87-100 Toruń, Poland

40 Department of Physics, University of the Free State, PO Box 339, 9300 Bloemfontein, South Africa

41 Charles University, Faculty of Mathematics and Physics, Institute of Particle and Nuclear Physics, V Holešovičkách 2, 18000 Prague 8, Czech Republic 